\title{
Benign Prostatic Hyperplasia in African American Men Merits Greater Consideration in Academic Medicine
}

\section{Joseph Wiedemer MS*\#, Trevor McKoy MS* and Michael Yu MD}

Wright State University Boonshoft School of Medicine, USA

\#These authors contributed equally to this work and are considered to be co-first authors

*Corresponding author: Joseph Wiedemer MS, Wright State University Boonshoft School of Medicine, USA, Tel: 410-271-6438; Email: Wiedemer.2@wright.edu

\section{Opinion}

Volume 4 Issue 2

Received Date: July 09, 2021

Published Date: July 20, 2021

DOI: $10.23880 /$ aabsc- 16000164

\section{Abstract}

Benign Prostatic Hyperplasia (BPH) is a non-cancerous enlargement of the prostate gland, which is highly prevalent in aging men. This process of benign prostate enlargement is known to occur more rapidly in African American men, which has resulted in a disproportionately increased prevalence relative to the general population. In the United States, the cause of this disproportionately increased risk in African Americans is believed to be due to both genetic factors as well as a complex myriad of variables related to health disparities. Despite a robust increase in research related to prostate cancer in African American men, the issue of BPH/LUTS in the African American community deserves equally increased attention in academic medicine. The purpose of this article is to provide a brief review of the medical literature on BPH/LUTS in the African American community to highlight unknown components of the disease and to demonstrate the need for more equitable urologic healthcare.

Keywords: Benign Prostatic Hyperplasia; Lower Urinary Tract Symptoms; Urology; Racial Disparities; Health Disparities; African American Health Outcomes; IPSS

Abbreviations: BPH: Benign Prostatic Hyperplasia; LUTS: Lower Urinary Tract Symptoms; DHT: Dihydrotestosterone; SNPs: Single-Nucleotide Polymorphisms; IPSS: International Prostate Symptom Score; AUA: American Urological Association; QOL: Quality of Life; IPSS: International Prostate Symptom Score.

\section{Introduction}

Globally, the prevalence of BPH among men is estimated at $8 \%$ by the age of 50 and $80 \%$ by the age of 90 [1-6]. Although BPH distinctively differs from prostate cancer, the exact underlying pathophysiology of $\mathrm{BPH}$ and its relation to prostate cancer is not fully understood. BPH is largely responsible for the exacerbation of lower urinary tract symptoms (LUTS) in aging men, which includes increased frequency of urination, urgency of urination, weakened urinary stream, nocturia, intermittency and urinary retention.

Inequalities in healthcare have existed for centuries and have caused imbalanced outcomes in overall morbidity and mortality among the African American community. It is clear that those with lower socioeconomic status may have less access to quality healthcare in the United States. Such inequalities have become particularly apparent over the past year amidst the COVID-19 pandemic and particularly within communities with large minority populations, poorer socioeconomic backgrounds and resource deprivation. 
It is important to note that LUTS caused by other conditions beyond BPH such as overactive bladder also significantly impact African Americans. A recent study in 2021 showed that the prevalence of urinary incontinence for men age 60-90 is consistently highest among African Americans relative to all other racial groups [7]. A 2020 study utilizing the Healthcare Cost and Utilization Project State Emergency Department Databases in Florida between 2005 and 2015 showed that African Americans are at increased risk for presentation to the ED for acute urinary retention (OR 1.15, $\mathrm{P}<0.001$ ) [8]. The observed risk for acute urinary retention was even greater for Hispanics (OR 1.75, $\mathrm{p}<0.001$ ) [9]. Given the likelihood that acute urinary retention is often caused by progressed and inadequately treated BPH, the results of this study support the likelihood that patients in these racial groups are relatively underdiagnosed and/or undertreated for BPH relative to white populations.

The failure to appropriately address BPH in African Americans is concerning on a myriad of levels, especially given the abundant evidence that this disease is linked to broader health conditions including cardiovascular disease $[1,6,9]$. A recent study in 2019 showed that African American men aged 35-49 with uncontrolled hypertension are 56\% more likely to have nocturia compared to African American men in the same age group with normotension [10]. BPH in its authentic sense is a histological diagnosis, which is not specifically pursued in the current standard of care. While other diseases associated with aging such as heart disease, cancer and diabetes are based on a combination of objective testing in addition to subjective patient recording, the diagnosis of $\mathrm{BPH}$ is almost entirely rooted in a clinical diagnosis reliant on subjective patient reporting in addition to a digital rectal exam. Since the initial diagnosis of BPH is highly subjective and African Americans may be more susceptible to various forms of reporting biases, further investigation pertaining to the reasons why specific populations are at risk should be pursued with considerable agency.

Historically, research regarding the effectiveness of diagnosis and treatment of benign prostatic hyperplasia have focused predominantly on white populations. However, African American men are disproportionately at higher risk for developing both BPH as well as prostate cancer [1,7]. Unfortunately, African Americans are also more likely to have poorer healthcare outcomes overall relative to Caucasians, which is largely attributed to issues related to healthcare access $[1,7,8]$. Physicians and healthcare stakeholders bear the responsibility to help mend the exposed social disparities and healthcare wounds. In the broader conversation of racial disparities in healthcare, we aim to raise awareness to this specific realm of urological health in men.

\section{Obesity and Metabolic Risk Factors}

There is a well-established linear correlation between obesity and BPH/LUTS severity thought to be mediated primarily by increased inflammation and hormonal changes related to obesity [11-14]. Higher body mass indexes have been shown to correlate with increased prostate volume, earlier onset of $\mathrm{BPH}$, and greater likelihood of requiring surgical intervention for effective BPH management [13]. It is also likely that co-morbidities related to obesity such as diabetes and hypercholesterolemia impact BPH progression [12]. The Baltimore Longitudinal Study of Aging observed an average $0.41 \mathrm{~mL}$ increase in prostate volume per every $1 \mathrm{~kg} /$ $\mathrm{m}^{2}$ increase in BMI [6]. Interestingly, multiple studies have demonstrated that physical activity significantly decreases the risk of symptomatic BPH [13].

The connection between BPH and obesity is particularly concerning as non-Hispanic black adults have the highest age-adjusted prevalence of obesity in the United States at $49.6 \%$ compared to other racial groups including nonHispanic white adults at $42.2 \%$ [15]. The impact of obesityrelated hormonal changes on BPH in African Americans is unclear given the unique androgenic polymorphisms believed to account for some of the differences in BPH presentation compared to other racial groups $[14,16]$. In addition to obesity-related increases in aromatase, multiple studies have shown that there is also a corresponding upregulation of estrogen receptors in prostatic tissue $[14,16]$. It is unclear how this understanding of $\mathrm{BPH}$ pathophysiology might impact the standard of care for African Americans or the general population.

Vitamin D insufficiency, which is often seen in African Americans, has been extensively studied as an exacerbating factor for LUTS [17]. A 2021 meta-analysis of 23 studies showed that Vitamin D insufficiency was associated with a 1.37-2.06-fold increased likelihood of having LUTS [17]. African Americans are at greater risk of Vitamin D deficiency due to decreased UV absorption secondary to higher skin melanin content. Some studies have observed decreased rates of endocytosis-mediated Vitamin D uptake of prostatic tissue in African American men, but this remains under active investigation $[18,19]$. The impact of metabolic risk factors such as obesity and nutritional deficiencies is a reflection of the complexity of BPH pathophysiology and the reality that these observed racial disparities are likely implicated by both social and genetic factors. More research is needed to quantify the impact of positive and necessary improvements in sociocultural variables on the disproportionate burden of $\mathrm{BPH}$ on minority communities. 


\section{Genetic Markers and Variability}

There has been extensive research on genetic idiosyncrasies that may partially account for the observed disparity of African American men in respect to prostate cancer, but relatively less in the context of BPH. Some have suggested that such genetic variations do not significantly contribute to the increased prevalence and corresponding disparities in African Americans [1]. Nonetheless, discerning the specifics of genetic variation and its impact on $\mathrm{BPH}$ pathophysiology remains an important area of investigation for the purposes of developing more effective diagnostic approaches and equitable treatment guidelines.

In respect to prostate cancer, there has been extensive research showing genetic polymorphisms impacting androgen receptors, androgen conversion to dihydrotestosterone (DHT), androgen synthesis, androgen deactivation, and signal transduction in African American men $[20,21]$. However, it is generally recognized that these genetic variations are also likely implicated in BPH pathophysiology [20]. In a recent study focused specifically on BPH, there was a significant increase in estrogen receptor and AKR1C1-which metabolizes DHT-expression in African Americans.

A univariate analysis of 620 healthy African American men identified two single-nucleotide polymorphisms (SNPs) associated with LUTS severity [22]. Specifically, rs5945572 was strongly associated with increased LUTS severity (OR=1.33, 95\% C.I. 1.04-1.71), while rs 445114 correlated with decreased symptom severity $(\mathrm{OR}=0.78$; 95\% C.I. 0.60-1.00). Another study identified two SNPs of anti-inflammatory interleukin 10 (IL-10) associated with increased LUTS severity and prostate cancer risk in African American smokers [4].

\section{Management of BPH}

Despite an increased prevalence of $\mathrm{BPH}$, the literature has consistently shown that African Americans are undertreated for BPH. Multiple studies have also shown that non-Caucasian men are at an increased risk of postoperative morbidity and mortality following surgical intervention for BPH [23-25]. The extent of disease progression at the time of BPH diagnosis should also be considered in the analysis of the frequency of surgery and subsequent complication rates among black men. However, accounting for BPH disease progression is admittedly challenging and unreliable in large-scale database analyses.

A retrospective study of 7 million men in the California Medi-Cal database showed that black men were 28.6\% less likely to receive medical treatment within the first year of diagnosis compared to patients of other races [24]. Interestingly, black men were less likely to receive surgical intervention for BPH (OR 0.83, $\mathrm{p}<0.05$ ) while Hispanic men were the most likely to receive surgery $(\mathrm{OR}=1.5, \mathrm{p}<0.001)$ relative to all other races [24]. The disproportionate utilization of surgery for BPH in African American men has been shown in other studies, even when controlling for socioeconomic variables, which was most recently demonstrated by Antoine, et al. [25,26]. However, a larger scale national study of the Southeastern US reported that African Americans were more likely to receive surgical intervention for BPH compared to Caucasians after adjusting for age, income, and insurance coverage (12.9\% vs. 9.1\% respectively, OR=1.65, CI 1.10-2.48) [27].

Treatment guidelines for conditions such as hypertension and heart failure suggest specific medications according to patient race designed to maximize treatment outcomes. Unfortunately, such racially-tailored treatment guidelines do not yet exist for the management of BPH and LUTS. It is unclear as to whether African Americans demonstrate a different response to first-line pharmacotherapy relative to other racial groups. Nonetheless, the existence of varying responses to medication by racial groups appears possible. For example, there has been extensive research into the incidental reduction in bladder cancer risk among patients using finasteride [28]. A large-scale study in 2017 affirmed this association in Caucasians and Hispanics, but showed that the same apparent protective effects of finasteride were not observed among African Americans [28]. It is also unclear as to whether African Americans would benefit from earlier surgical intervention.

\section{Screening, Literacy, and Community Intervention}

LUTS are commonly reported using the International Prostate Symptom Score (IPSS) which is an 8-question written screening tool developed in 1992 by the American Urological Association (AUA), for both the initial evaluation of BPH and monitoring treatment efficacy [9]. The survey is practical in that it both quantitatively analyzes symptoms of BPH and enables patients to disclose symptoms with a degree of privacy, as it is almost always self-administered. It contains seven questions related to BPH symptoms and one question related to the patient's perceived quality of life. BPH symptom severity is proportionate to the IPSS score, which ranges from 0 to 35 and categorizes symptoms as mild (17), moderate (8-19), or severe (20-35). The IPSS survey is widely used in Urology and the AUA also encourages its use in primary care settings. Furthermore, the survey is widely utilized in BPH research. The final question on the survey is a quality of life (QOL), also known as a "bother score" to gauge the patient's desire to pursue treatment. 
Unfortunately, the medical literature consistently supports the concerning reality that African American men are insufficiently screened for both BPH and prostate cancer [29]. Consistent with other healthcare issues, African American men are more likely to be disproportionately impacted by insufficient healthcare literacy [30-32]. Interestingly, previous studies have shown that black men are more likely to report a lower bother score relative to white populations with similar LUTS severity [33]. This difference was even more prominent in black men with lower social support and/or emotional health [33]. It is unclear exactly how this insight could be utilized to improve the diagnosis and treatment of BPH in African American men, but further research into considering lower bother score thresholds for treatment initiation should be considered.

The utilization of healthcare education in black-owned barbershops has been extensively researched over the last decade with impressive increases observed in prostate cancer screening [34-36]. However, it is unclear if such an intervention has been studied in the context of BPH/ LUTS. Deliberate interventions that address health literacy in respect to prostate cancer have been shown to enhance shared decision making in African American Men [30,37]. Generally, the authors believe that research regarding prostate cancer in African American men may be helpful reference in discerning more effective BPH treatment, especially in areas where analogous research is lacking.

\section{Conclusion}

The authors firmly believe that Men's Health in the African American community should receive substantial investments in healthcare research by the academic community. Furthermore, members of the healthcare community should facilitate qualitative resolution by building stronger relationships between physicians and members in underserved communities. Future studies on LUTS and BPH should aim to employ larger sample sizes and control for confounding variables such as family history, smoking, and obesity. Moreover, primary care providers should strongly consider routinely administering the IPSS survey to all of their male patients over 50, especially African Americans.

It is clear that the evaluation and treatment of LUTS is highly complex and should be uniquely approached in the African American community. Current BPH treatment guidelines from the AUA do not separately address the unique circumstances of African American men. The authors firmly believe that the formulation of specially tailored treatment guidelines should be investigated with considerable urgency. As members of the medical profession, we aim to advocate and champion attempts to achieve equitable urologic health in the African American community.

\section{Acknowledgements}

The co-first authors would like to thank their mentor Daniel Pucheril MD for reviewing this manuscript and providing his expert perspective on Urological care.

\section{Disclosure Statement}

Each of the authors declare that they have no known competing financial interests, employment history, personal relationships, or competing interests of any form current/existing that could have appeared to influence this manuscript.

\section{References}

1. Fowke JH, Munro H, Signorello LB, Blot WJ, Penson DF (2011) Association between socioeconomic status (SES) and lower urinary tract symptom (LUTS) severity among black and white men. J Gen Intern Med 26(11): 13051310.

2. Van Den Eeden SK, Shan J, Jacobsen SJ, Aaronsen D, Haque R, et al. (2012) Evaluating Racial/Ethnic Disparities in Lower Urinary Tract Symptoms in Men. J Urol 187(1): 185-189.

3. Daskivich TJ, Ryan G, Khodyakov D, et al. (2020) General \& Epidemiological Trends \& Socioeconomics: Practice Patterns, Quality of Life and Shared Decision Making IV. J Urol 203(4): 1.

4. DeSantis CE, Miller KD, Goding Sauer A, Jemal A, Siegel RL (2019) Cancer statistics for African Americans, 2019. CA Cancer J Clin 69(3): 211-233.

5. Abbas M, Mason T, Ibad A, Khraiwesh M, Apprey V, et al. (2020) Genetic polymorphisms in IL-10 promoter are associated with smoking and prostate cancer risk in African Americans. Anticancer Res 40(1): 27-34.

6. Lim K Bin (2017) Epidemiology of clinical benign prostatic hyperplasia. Asian J Urol 4(3): 148-151.

7. Akbar A, Liu K, Michos ED, Brubaker L, Markossian T, et al. (2021) Racial Differences in Urinary Incontinence Prevalence, Overactive Bladder and Associated Bother among Men: The Multi-Ethnic Study of Atherosclerosis. J Urol 205(2): 524-531.

8. Patel PM, Sweigert SE, Nelson M, Gupta G, Baker M, et al. (2020) Disparities in benign prostatic hyperplasia progression: Predictors of presentation to the emergency department in urinary retention. J Urol 204(2): 332-336. 
9. Ostfeld RJ, Allen KE, Aspry K, Brandt EJ, Aaron Spitz, et al. (2020) Vasculogenic Erectile Dysfunction: The Impact of Diet and Lifestyle. Am J Med 134(3): 310-316.

10. Victor RG, Li N, Blyler CA, Mason OR, Chang LC, et al. (2019) Nocturia as an Unrecognized Symptom of Uncontrolled Hypertension in Black Men Aged 35 to 49 Years. J Am Heart Assoc 8(5): e010794.

11. Penson DF, Munro HM, Signorello LB, Blot WJ, Fowke JH, et al. (2011) Obesity, Physical Activity and Lower Urinary Tract Symptoms: Results From the Southern Community Cohort Study. JURO 186(6): 2316-2322.

12. Lee SH, Lee SK (2017) Does Race/Ethnicity Have a Role in a Link between Lower Urinary Tract Symptoms and Metabolic Syndrome? - European Medical Journal. Eur Med J 2(1): 69-75.

13. Parsons JK, Sarma A V, McVary K, Wei JT (2013) Obesity and benign prostatic hyperplasia: Clinical connections, emerging etiological paradigms and future directions. J Urol 189(1s): S102-106.

14. Liu T, Ricke E, Strand D, Dhir R, Ricke W (2020) mp06-04 steroid hormone metabolism mediated racial disparity in men with benign prostatic hyperplasia. J Urol 203(4s): e52-e52.

15. (2021) Adult Obesity Facts. Overweight \& Obesity. CDC.

16. Wang Z, Xue B, Wu S, Hu L, Tabatabaei S, et al. (2020) mp06-03 obesity-associated inflammation induces androgenic to estrogenic switch in the prostate gland. J Urol 203(4s): e52-e52.

17. Yuan P, Wang T, Li H, Lan R, Li M, et al. (2020) Systematic Review and Meta-Analysis of the Association between Vitamin D Status and Lower Urinary Tract Symptoms. J Urol 205(6): 1584-1594.

18. Richards Z, Nonn L, Gann PH (2018) The Free Hormone Hypothesis and Ancestry-Related Differences in Prostate Vitamin D Uptake and Metabolism. University of Illinois at Chicago.

19. Nonn L (2017) Disparate Vitamin D Activity in the Prostate of Men with African Ancestry. Defense Technical Information Center.

20. Hatcher D, Daniels G, Osman I, Lee P (2009) Molecular mechanisms involving prostate cancer racial disparity. Am J Transl Res 1(3): 235-248.

21. Omri N, Kamil M, Alexander K, Alexander K, Edmond S, et al. (2020) Association between PSA density and pathologically significant prostate cancer: The impact of prostate volume. Prostate 80(16): 1444-1449.
22. Helfand BT, Loeb S, York N, Reinhardt D, Cooper PR, et al. (2013) 1740 genetics variants that increase severe lower urinary tract symptoms in african-american men. J Urol 189(4): 715.

23. Ngampoopun $M$, Suwanpakdee P, Jaisupa N, Nabangchang C (2018) Effectiveness and Adverse Effect of Intravenous Lacosamide in Nonconvulsive Status Epilepticus and Acute Repetitive Seizures in Children. Neurol Res Int 2018: 8432859.

24. Gill H (2015) Racial Disparities in the Treatment of Benign Prostatic Hyperplasia. Med Surg Urol 4(4): 157.

25. Mithal A, Singh G, Mannalithara A, Malani R, Triadafilopoulos G, et al. (2010) 1790 health care disparities in medical and surgical treatment of benign prostatic hyperplasia. J Urol 183(4): 694.

26. Antoine S, Carmichael H, Lloyd G (2020) pd40-04 the impact of race, ethnicity and insurance status on surgery rates for benign prostatic hyperplasia. J Urol 203(4): 816.

27. Fowke JH, Murff HJ, Signorello LB, Lund L, Blot WJ (2008) Race and Socioeconomic Status are Independently Associated With Benign Prostatic Hyperplasia. J Urol 180(5): 2091-2096.

28. Srivastava A, Fram E, Agalliu I, Schoenberg M, Sankin A (2017) pd57-07 race and finasteride use: differential impact on bladder cancer risk. J Urol 197(4): 1123-1124.

29. Lu CD, Adeyemi O, Anderson WE, Hetherington TC, Slawson DC, et al. (2021) Racial Disparities in PSA Screening and Referral to Urology in a Large, Integrated Healthcare System: A Retrospective Cohort Study. J Urol 206(2): 270-278.

30. Oliver JAS, Allen RS, Eichorst MK, Mieskowski L, Ewell PJ, et al. (2018) A pilot study of prostate cancer knowledge among African American men and their health care advocates: implications for screening decisions. Cancer Causes Control 29(7): 699-706.

31. Posid T, Amin S, Khalil K, Sourial M, Sharp D, et al. (2020) pd40-08 exploring barriers to healthcare in underserved minority populations in central ohio. J Urol 203(4): 818.

32. Kilbridge K, Martin DW, Filson C, Trinh QD, Williams S, et al. (2020) pd40-07 addressing health literacy improves shared decision making in underserved african american prostate cancer patients. J Urol 203(4s): 817-818.

33. Men N, Kellogg Parsons J, Bergstrom J (2008) Psychosocial factors related to bother in African american men: the flint men's health study.

34. Luque JS, Roy S, Tarasenko YN, Ross L, Johnson J, et al. 
(2015) Feasibility Study of Engaging Barbershops for Prostate Cancer Education in Rural African-American Communities. J Cancer Educ 30(4): 623-628.

35. Frencher SK, Sharma AK, TeklehaimanotS, Wadzani D, Ike JE, et al. (2016) PEP Talk: Prostate Education Program, Cutting Through the Uncertainty of Prostate Cancer for Black Men Using Decision Support Instruments in Barbershops. J Cancer Educ 31(3): 506-513.
36. Luque JS, Ross L, Gwede CK (2016) Prostate cancer education in African American barbershops: Baseline client survey results and differences in decisional conflict and stage of decision making. Am J Mens Health 10(6): 533-536.

37. Schoenfeld ER, Francis LE, Adler HL, DeGrasse A, Phillips JP, et al. (2008) prostate cancer knowledge in an african american community. J Urol 179(4): 162. 